\title{
A NOTE FOR BOUNDS OF NORMS OF HADAMARD PRODUCT OF MATRICES
}

\author{
RAMAZAN TÜRKMEN AND DURMUŞ BOZKURT
}

\begin{abstract}
In this paper, we have established upper bounds for the spectral norms of CauchyToeplitz matrix and Cauchy-Hankel matrix, with $g=1 / 2$ and $h=1$. Moreover, we have obtained an upper bound for the spectral norm of Hadamard product of Cauchy-Toeplitz and Cauchy-Hankel matrices. In addition, we have established an upper bound for the norm of Hadamard product of Cauchy-Toeplitz and Cauchy-Hankel matrices.
\end{abstract}

Mathematics subject classification (2000): 15A18, 15A45, 15A57, 15A60.

Key words and phrases: Matrix norm, Cauchy-Toeplitz matrices, Cauchy-Hankel matrices, spectral norm, $\ell_{p}$ norm, singular values, Hadamard product.

\section{REFERENCES}

[1] R. A. Horn And C. R. Johnson, Matrix Analysis, Cambridge University Press, New York, (1985).

[2] R. A. Horn And C. R. Johnson, Topics in Matrix Analysis, Cambridge University Press, New York, (1991).

[3] T. Ando, Roger A. Horn And Charles R. Johnson, The Singular Values of a Hadamard Product: A Basic Inequality, Linear and Multilinear Algebra, 21, (1987), 345-365.

[4] R. MAthias, The Spectral Norm of a Nonnegative Matrix, Linear Algebra Appl., 131, (1990), $269-284$.

[5] D. BozKurT, On the $\ell_{p}$ Norms of Cauchy-Toeplitz. Matrices, Linear and Multilinear Algebra, 44, (1998), 341-346.

[6] D. BozKuRT, On the $\ell_{p}$ Norms of Cauchy-Hankel Matrices, Journal of Scientific Research Foundation, 1, (1996), 27-32.

[7] S. V. PARTER, On the Distribution of Singular Values of Toeplitz Matrices, Linear Algebra Appl. 80, (1986), 115-130.

[8] D. Bozkurt, On the Bounds of Cauchy-Toeplitz, Matrices, Sib. J. Diff.Equation, 3, (1996), $135-142$.

[9] E. E. TyrtyshniKov, Cauchy-Toeplitz matrices and Some Applications,Linear Algebra Appl., 149, (1991), 1-18. 